\title{
DOES THE DIELECTRIC-DIELECTRIC INTERFACE AFFECT THE POSITRON DIFFUSION CURRENT?*
}

\author{
J. BeLICZyŃSKI, E. DĘBowsKa AND W. ŚWIĄTKowsKi \\ Institute of Experimental Physics, Wrocław University \\ Pl. Maxa Borna 9, 50-204 Wrocław, Poland
}

The angular correlations of annihilation radiation have been measured for $\mathrm{NaI}, \mathrm{NaF}$ and $(\mathrm{NaI})_{0.65}(\mathrm{NaF})_{0.35}$ alloy. The angular correlations of annihilation radiation for the alloy appeared to be more similar to the one for $\mathrm{NaI}$ than it should be expected from the content of $\mathrm{NaI}$ in the alloy. The result can be easily explained by assuming that $\mathrm{NaI} / \mathrm{NaF}$ interface acts directionally on positron diffusion movement.

PACS numbers: $68.35 . \mathrm{Wm}$

\section{Introduction}

- Positrons from a radioactive source entering a sample are slowed down and preliminary (prior to the diffusion) implanted according to the absorption law. When the sample consists of different materials whose pieces are small as compared with the positron absorption ranges in them, the fraction of the positrons implanted in each material is proportional to the weight percentage of the material in the sample. Thus the positron annihilation characteristics for such a sample should be the weighted average of each material characteristics.

If the pieces are of the positron diffusion length order, the positron interaction with material interface is able to change the situation. Światkowski et al. [1], for example, observed that the metal-metal interface may act directionally on positron diffusion current. The angular correlation of positron annihilation radiation (ACAR) for multi-thin-layer bimetallic sample appeared to be closer to the ACAR for one metal than it resulted from its content in the sample.

The ACAR and positron lifetime measurements for similar metal-dielectric sample indicated the possibility of positron trapping at the interface of such a kind $[2,3]$. These two effects: directional action of metal-metal contact and positron trapping at metal-dielectric interface can be expected from the analysis of the positron potential energy shape in the vicinity of the interface.

*The work was supported by the Ministry of National Education within the research project $105 /$ E-319/92. 
Lewowski et al. [4] and Mazur [5] observed that some dielectric-dielectric interfaces (between two ionic crystals) could accelerate the electrons passing through them. The results suggested that such interfaces are likely to act directionally on the positrons as in the case of metal-metal contact. The results indicating that such an expectation can be a reality, are presented in the paper.

\section{Experiment}

The object of our study was an alloy consisting of $65 \mathrm{wt} . \% \mathrm{NaI}$ and of $35 \mathrm{wt} . \%$ $\mathrm{NaF}$. Such contents of alloy components were chosen because due to them the alloy is an eutectic one. It means that it can be treated as a "mixture" of closely contacting $\mathrm{NaI}$ and $\mathrm{NaF}$ grains. The alloy was obtained by melting appropriate amounts of $\mathrm{NaI}$ and $\mathrm{NaF}$ in vacuum. For the alloy as well as for $\mathrm{NaI}$ and $\mathrm{NaF}$ samples there were measured ACARs using standard 1D apparatus with $0.8 \mathrm{mrad}$ angular resolution. The samples and the ${ }^{22} \mathrm{Na}$ source were situated, during the ACAR measurements, in vacuum chamber $\left(\approx 10^{-4} \mathrm{~Pa}\right)$. For each ACAR the count number at maximum was close to 40000 . The ACARs obtained are presented in Fig. 1.

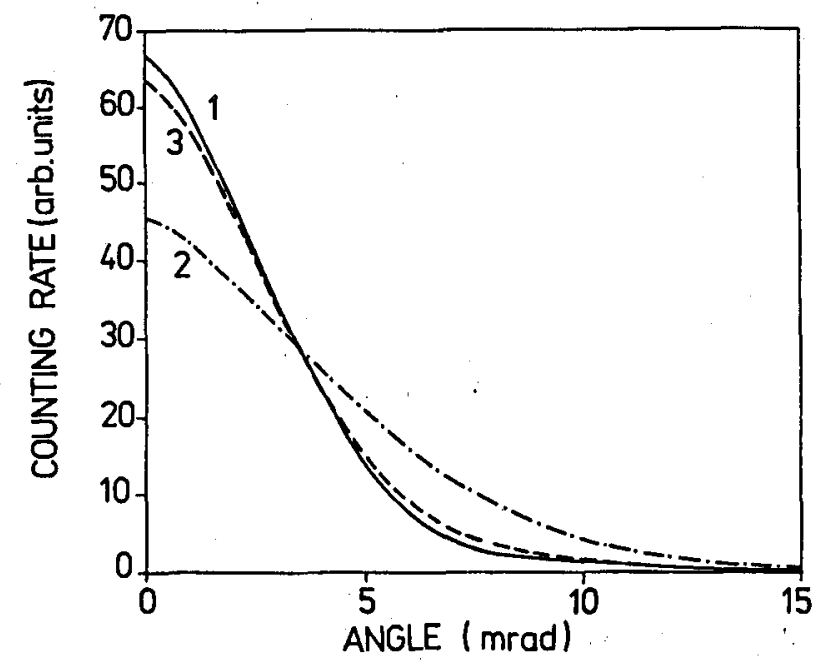

Fig. 1. Angular correlation of annihilation radiation curves for $\mathrm{NaI}(1), \mathrm{NaF}$ (2) and $(\mathrm{NaI})_{0.65}(\mathrm{NaF})_{0.35}$ alloy (3) normalized to the same area. The statistic errors are smaller than $0.5 \%$ of maximum counting rates.

\section{Conclusions}

From the analysis of the ACARs presented in Fig. 1 it follows that the ACAR for the alloy can be well approximated by the sum of $0.85 \mathrm{ACAR}$ related to $\mathrm{NaI}$ and 
of 0.15 ACAR related to $\mathrm{NaF}$. This may be easily explained if one assumes that $85 \%$ of positrons annihilated in $\mathrm{NaI}$ grains and the rest of them (15\%) in $\mathrm{NaF}$ ones. However, because the alloy consits of $65 \mathrm{wt} . \% \mathrm{NaI}$ and $35 \mathrm{wt} . \% \mathrm{NaF}$, the fraction of positrons primarily stopped in $\mathrm{NaI}$ grains was (according to absorption law) equal to 0.65 , and the one stopped in $\mathrm{NaF}$ grains to 0.35 . Thus, in order to explain the shape of ACAR for the alloy one should assume that approximately $50 \%$ of positrons primarily stopped in $\mathrm{NaF}$ were able to pass, before the annihilation, to $\mathrm{NaI}$ with no possibility for positrons to pass from $\mathrm{NaI}$ to $\mathrm{NaF}$. Such an explanation of the effect observed is in agreement with the expectation that the interface may act directionally on the positron movement. Two other aspects relevant to the issue should be taken into account, however:

(1) According to Lewowski and Mazur $[4,5]$ there exists a potential energy jump for electron at the interface between two ionic crystals. This is due to the double charged layer caused by the reordering of the ions in the vicinity of the interface. We have assumed that the layer should give the potential energy jump also for another particle, positron in particular. But we should mention at the moment that the potential energy jump for a charged particle, when going from one of the two dielectrics being in contact to the other one, could be also connected with the difference in their dielectric properties. Let us consider the positron inside the dielectric. In this case the electric field produced by the positron should be different from the one for the positron in vacuum. Because a positron can be treated approximately as a point charge, the electric field in its vicinity inside the dielectric should be the same as it is in the vacuum. However, for the distances greater than approximately a half of the lattice constant (say $R$ ) the field in the dielectric should be weeker by the factor $\varepsilon$ ( $\varepsilon$ is the dielectric constant). The electric field energy attributed to the whole volume outside the sphere of radius $R$ equals

$$
U_{\mathrm{v}}=\frac{e^{2}}{8 \pi \varepsilon_{0} R}
$$

for vacuum, and

$$
U_{\mathrm{d}}=\frac{e^{2}}{8 \pi \varepsilon_{0} \varepsilon R}
$$

for dielectric. Thus the potential energy jump for the positron going from vacuum to dielectric should be expected to be of the order of

$$
\Delta U_{\mathrm{v}, \mathrm{d}}=U_{\mathrm{v}}-U_{\mathrm{d}}=\frac{e^{2}}{8 \pi \varepsilon R}(1-1 / \varepsilon),
$$

while for the positron going from the one dielectric (say 1) to the other one (say 2) of the order of

$$
\Delta U_{1,2}=\frac{e^{2}}{8 \pi \varepsilon_{0}}\left[\frac{\varepsilon_{1}-1}{R_{1} \varepsilon_{1}}-\frac{\varepsilon_{2}-1}{R_{2} \varepsilon_{2}}\right] .
$$

Taking $R$, in the formula (1), being equal to $0.1 \mathrm{~nm}$ one obtains $U_{\mathrm{v}}=0.72 \mathrm{eV}$. For $\mathrm{NaI}$ and $\mathrm{NaF}$ the lattice constants equal 0.647 and $0.462 \mathrm{~nm}$, and dielectric constants 6.6 and 6.0 , respectively. For these values the formula (4) gives the value $0.071 \mathrm{eV}$ with the direction of the jump preventing the positron transfer from $\mathrm{NaF}$ to NaI, which is not consistent with the reported effect. One should however have 
in mind that the estimations based on the formula (4) are very simplified ones, and that the effective energy jump felt by the positron may be the balance of the jumps resulting from both the reordering of the ions in the vicinity of the interface and the difference of dielectric properties of the sample materials.

(2) The preferential annihilation in one of the materials being in contact could be caused by the differences between positron diffusion ranges (positron diffusion lengths) and positron lifetimes for these materials. Thus the explanation of the effect observed in our experiment should be treated only as one of different possibilities. One should also take into account that the ACAR for the alloy could be strongly affected by the possibility of positron trapping at the grain boundary. The fact that the ACAR for the alloy can be well approximated by the sum of ACARs characteristic of the alloy components seems to indicate however that the annihilation takes place in the bulk part of the grains mainly.

It should be mentioned that our earlier, similar studies for KCl-KF system did not indicate the effect reported here [6].

\section{References}

[1] W. Świątkowski, B. Rozenfeld, H.B. Kołodziej, S. Szuszkiewicz, Acta Phys. Pol. A 54, 79 (1975).

[2] R. Ewertowski, W. Świątkowski, Phys. Scripta 35, 746 (1987).

[3] R. Ewertowski, W. Świątkowski, Phys. Status Solidi A 102, 555 (1987).

[4] T. Lewowski, P. Mazur, Acta Univ. Wratisl. Mat. Fiz. Astron. 48, 177 (1986).

[5] P. Mazur, Surf. Sci. 231, 95 (1990).

[6] E. Dębowska, W. Świątkowski, in: Proc. of the 6th Conf. Surf. Phys., Eds. J. Ledzion, Z. Fijarczyk, University of Łódź, Lódź 1992, p. 142. 